\title{
RECHERCHES SUR LA TENEUR EN CHLORURES DU LAIT ET SA VALEUR DANS LE DIAGNOSTIC DES DIVERSES MAMIMITES BOVINES ET CELUI DU LAIT PATHOLOGIQUE (1)
}

\author{
par \\ Docteur DJ. FILIPOVITCH \\ Service Vétérinaire fédéral - Belgrade \\ et \\ Mme Darinka FILIPOVITCH \\ Pharmacienne
}

Le dosage de la teneur en chlorures du lait a pour but de déceler les diverses mammites, et en particulier, des mammites chroniques chez les femelles laitières. Par conséquent, il peut servir dans une certaine mesure, au dépistage du lait pathologique.

En réalité, c'est seulement une des méthodes pour ces diagnostics. Dans l'application pratique il existe deux techniques. Tout d'abord la technique analytique effectuée uniquement dans les laboratoires par le dosage quantitatif des chlorures des cendres ou le dosage direct dans le lait, et ensuite la méthode rapide, qualitative, qui indique seulement si la teneur en chlorure est supérieure à $0,14 \%$.

Depuis 1946, et pendant les dix dernières années, nous avons fait des recherches sur la teneur en chlorures du lait afin de vérifier sa valeur dans le diagnostic des diverses mammites bovines. En même temps, nous avons fait l'analyse complète du lait de 9.086 vaches laitières, provenant de diverses fermes agricoles, principalement en Serbie, en Croatie, sur un nombre limité, ainsi qu'en Bosnie et en Hercégovine.

Nous avons utilisé une des trois techniques rapides recommandée par C. E. HAYDen et Rosell [2], effectuées de façon suivante : on prend $5 \mathrm{~cm}^{3}$ d'une solution de nitrate d'argent à 1 gr. 3.415 par litre d'eau, puis on ajoute 2 gouttes d'une solution de bichromate de potassium à $10 \%$ et l'on ajoute à ce mélange $1 \mathrm{~cm}^{3}$ de lait. Si la teneur en chlore du lait est de $0,14 \%$ ou supérieure à ce chiffre, il se développe pendant environ une minute une coloration jaune. Comme la méthode est extrêmement simple et donne de bons résultats, nous avons fait par ce procédé la détermination de la teneur en chlorure du lait aussi bien au laboratoire que dans les fermes.

On considère que la teneur en chlore calculée en milligrammes

(1) D'après un Rapport présenté au VII e Congrès international de Pathologie comparée (Lausanne, mai 1955). 
de $\mathrm{Cl}$ par 100 grammes de lait est généralement comprise entre 70 et 130 , quoiqu'on considère normale jusqu'à 140 milligrammes et seulement tel chiffre et au-dessus comme pathologique. Il nous paraît préférable de nous exprimer en pourcentage car c'est plus pratique, par exemple : $0,07 \%$ à $0,14 \%$.

W. L. Davis [3], sur 7.802 échantillons de lait, a constaté que la teneur en chlore variait chez les vaches de la race de Frise, de 0,11 à $0,12 \%$, tandis que la moyenne a été de $0,097 \%$ pour le lait des vaches Schorthorn et $0,093 \%$ pour les vaches de Guernesey.

J. W. Blood et A. Rowlands [4] ont étudié comparativement le dosage direct du chlore dans le lait par une solution de nitrate d'argent, donc par l'essai rapide et des analyses précises. D'après tous ces résultats ils considèrent que l'assertion de RoseLL selon laquelle le lait qui contient plus de $0,14 \%$ de chlore dénote la mastite chez l'animal qui a fourni le lait, ne semble pas justifiée. Ces auteurs ont constaté en effet, par des examens chimiques et bactériologiques, que des échantillons de lait ayant une teneur normale en chlore, comprise entre 0,07 et $0,12 \%$ peuvent contenir des streptocoques, et qu'au contraire, dans certains laits ayant une teneur supérieure à ces limites, la présence de mammite n'est pas toujours obligatoire.

D'après les résultats des travaux de l'Institut National de Recherches de laiterie de Reading, en 1936 [5], la teneur en chlore d'échantillons individuels des vaches du troupeau de l'Institut a été déterminée sur un total de 1.086 échantillons donnant une teneur moyenne de $0,094 \%$.

E. Pijanovski [6] a analysé 438 échantillons de lait de quartiers différents, provenant de vaches ayant des symptômes de mammite d'un type surtout latent et il a constaté une forte variation de la teneur en chlore jusqu'à $0,33 \%$. Cet auteur a trouvé une concordance marquée entre les résultats des tests du chlore, du $p \mathbf{H}$, de la teneur en leucocyte et un rapport très net entre les pourcentages de chlore et de lactose dans le lait.

Burkex [7] constate dans les cas d'infection streptococcique, d'autres éléments analytiques, une hausse du pourcentage de chlore qui dépasse $0,14 \%$ et, après la guérison, il note que le pourcentage de chlore diminue.

J. M. Moreira [8] trouve sur 267 vaches fortement șuspectes de mammite chronique que le test de chlorure est efficace dans $89,26 \%$ des cas.

\section{Interprétation des résultats d'analyse du lait}

Nous avons cité brièvement les résultats de ces quelques auteurs ayant traits caractéristiques et utiles en comparaison avec nos 
résultats, laissant de côté de nombreux autres travaux. Bien entendu nous n'avons pas eu à notre disposition toute la littérature concernant cette matière, surtout pendant les dernières années.

Dans l'interprétation de ces résultats des recherches sur les variations de la teneur en chlorures du lait, nous avons pris en considération le nombre d'échantillons du lait analysé, le nombre des cas à teneur élevée au-dessus de $0,14 \%$, puis les cas de laits normaux et anormaux dont les éléments constitutifs sont diminués au-dessous de la limite minimum du lait normal et qui ordinairement présentent les caractéristiques du lait de mammite, e'est-à-dire pathologique.

Il résulte de ces recherches, sur un total de 9.086 échantillons du lait de vaches laitières, que dans 1.646 échantillons de lait fut décelé un taux des chlorures supérieur à $0,14 \%$, soit $18,11 \%$. Ces résultats sont très différents selon les fermes. Certaines fermes ont le pourcentage favorable, les cas d'échantillons à la teneur de chlorure élevée n'atteignant pas $2 \%$, tandis qu'il y en a d'autres dont le pourcentage est très élevé : $25 \%, 30 \%, 40 \%$ et même supérieur à $50 \%$.

Les 1.646 échantillons de lait à taux de chlorures augmenté peuvent être partagés en deux groupes : l'une appartenant au lait anormal avec 322 échantillons, soit $19,56 \%$, et le deuxième groupe appartenant au lait normal avec 1.324 échantillons, soit $81,89 \%$. Voici donc une preuve incontestable de la véritable signification de la valeur de la détermination du taux des chlorures du lait dans le diagnostic des diverses mammites et du lait pathologique. Si on rencontre dans ces recherches seulement $19,56 \%$ des cas du lait anormal ayant la teneur en chlorure augmentée et à plus de $80 \%$ de lait tout à fait normal au point de vue physico-chimique, cela signifie en réalité qu'un taux de chlorure du lait supérieur à $0,14 \%$ ne signifie pas qu'il s'agit d'un lait mammitique ou pathologique.

Sur un certain nombre d'échantillons de lait, nous avons effectué le dosage du taux de chlorures par la méthode titrimétrique plus précise afin de pouvoir vérifier l'exactitude de nos essais simples et rapides, que nous considérons comme valables.

Il est très intéressant de remarquer que chez 1.344 vaches laitières de la ferme agricole "Belje », on a trouvé dans 345 cas du lait arec le taux de chlorure augmenté, dont 115 cas appartenant au lait anormal, soit $33,33 \%$, ou bien, par rapport au nombre total de 322 échantillons du lait anormal : $35,71 \%$.

Nous considérons que cette augmentation de pourcentage des cas avec le lait anormal et en chlorure augmenté doit être attribuée au fait que la mammite streptococcique a été très répandue chez les vaches laitières, ce que nous avons décelé immédiatement après les analyses effectuées dans cette ferme au début de nos recherches 
et au cours de l'année 1946. Répétons que, ordinairement, dans la, mammite on trouve la teneur en chlorure augmentée mais, comme on le voit, nous rencontrons beaucoup plus fréquemment la présence de chlorure augmenté dans le lait normal, sans prétention de pouvoir expliquer ici ces faits.

Sur 937 échantillons de lait au cours de l'année 1950, d'origine des Coopératives agricoles aux environs de Belgrade et sur le territoire des districts : Belgrade, Stara Pazova et Pancevo, nous avons constaté la teneur en chlorure augmenté sur 330 échantillons dont 96 appartenaient au lait anormal, soit $29,09 \%$.

Dans ces trois districts mentionnés plus haut avec 937 échantillons de lait examiné et dans la ferme "Belje " avee 1.344 échantillons, ce qui fait au total 2.281 vaches, nous avons décelé 675 cas du lait à chlorure augmenté $(41 \%$ ) tandis que sur un total de 47 autres fermes ayant 6.805 vaches laitières, on a constaté seulement 971 cas à chlorure augmenté (59\%).

Parmi les échantillons de la majorité du reste des vaches, on a trouvé environ $30 \%$ de cas d'échantillons de lait anormal.

Il faut expliquer que, dans ces trois districts, il ne s'agissait pas de vaches atteintes de mammite, mais de vaches mal logées à cause du manque d'étables hygiéniques (car ce fut le début de la création de ces coopératives), puis, insuffisamment nourries et soignées. Au contraire, sur l'ensemble d'un groupe de 30 fermes, comptant 3.866 vaches laitières, on a trouvé seulement 20 échantillons de lait anormal avec le taux de chlorure augmenté.

Il est d'importance de dire quelques mots au sujet du rapport, de concordance entre les pourcentages de lactose dans le lait et de chlore en teneur élevée. La plupart des vaches laitières ayant les symptômes de mammite aiguë ont fourni du lait à teneur normale, en chlorures et en sucre. Cependant on a trouvé plus de $20 \%$ de cas à chlorures augmenté et à teneur en sucre normale, ce que l'on rencontre ordinairement chez les vaches étant en fin de lactation. Nous avons constaté également dans environ $10 \%$ des cas, que l'augmentation de la teneur en chlorure a été suivie par la diminution de la teneur en sucre.

A la fin, nous constatons presque dans le même pourcentage de cas, une concordance entre la diminution de la teneur en chlorures et en sucre. Comme on le voit clairement, qu'il n'y a pas une règle absolue, dans tous les cas, des variations de la teneur de ces deux cristalloïdes dans le lait anormal pour diverses causes.

\section{Résumé et conclusion}

Depuis 1946 et pendant les dix dernières années, nous avons fait des recherches sur la teneur en chlorures du lait de 9.086 échan- 
, tillons provenant du même nombre de vaches laitières, en effectuant des analyses complètes du lait.

Le dosage qualitatif des chlorures a été effectué par la méthode rapide qui nous décèle seulement si le lait contient plus de $0,14 \%$ de $\mathrm{Cl}$, et sur un nombre assez limité par la méthode titrimétrique, afin de vérifier quelle est la valeur de ce dosage dans le diagnostic de diverses mammites et pour le dépistage du lait pathologique, ou anormal pour une raison quelconque.

Sur 9.086 échantillons nous en avons trouvé 1.646 avec la teneur en chlorures au-dessus de $0,14 \%$ dont 322 cas, soit $19,56 \%$ appartient au lait pathologique-anormal, tandis que le reste et la majorité de 1.324 échantillons ou $81,89 \%$ appartient au lait normal dans ses éléments chimiques.

On n'a pas constaté de concordance entre le pourcentage en teneur élevée de chlore et de lactose. De toutes ces recherches on peut conclure que :

10 Le dosage qualitatif et quantitatif des chlorures dans le lait peut être utilement pratiqué dans les analyses individuelles du lait, mais sans grande valeur comme on le prétend très souvent, pour le diagnostic de diverses mammites et le dépistage du lait pathologique ou anormal;

$2^{\circ}$ En plein accord avec BLoon et Rowlands, nos résultats montrent également que le lait qui contient plus de $0,14 \%$ de chlore, ne dénote pas la mammite chez les femelles laitières et aussi le lait ayant une teneur normale en chlorures, comprise entre 0,07 et $0,13 \%$ peut provenir de femelles atteintes de mammite, particulièrement de celles au stade aigu de l'évolution;

$3^{\circ}$ Dans la mammite chronique avec lésions des tissus mammaires, les femelles sécrètent un lait pathologique caractérisé dans la plupart des cas par la diminution de ses constituants physicochimiques, tandis que dans la mammite aiguë on obtient très souvent un lait tout à fait normal, sauf de la flore microbienne ;

$4^{\circ}$ On rencontre toutes sortes de variations dans la teneur en chlorures et en sucre du lait pathologique ou anormal, sans aucune règle absolue pour diverses causes.

\section{BIBLIOGRAPHIE}

[1] C. E. Hayden. Rept. N. Y. State Vet. Coll. Cornell. Univ., 1933, 55-58.

[2] J. M. Rosell. Le Lait, 14, 1934, no 138, p. 914 à $924 ; n^{\circ} 140$, p. 1050 à 1060 .

[3] W. L. Davis. Journal Dairy Res., 9, 1938, 327.

[4] J. W. Blood et Rolw Ands. Journal of Dairy Res., 7, no 1, 1936-1937.

[5] Annual Report Univ. of Read. Nat. Inst, for Res. in dairyng, 1936. 
[6] E. Pijanovski, Le Lait, no 167, 1937, p. 731. .

[7] BURKey. Le Lait, novembre 1937, p. 980.

[8] J. M. Moreira. Bulletin vétérinaire, n², 1938, p. 377. Lisbonne.

\title{
BIBLIOGRAPHIE ANALYTIQUE
}

\author{
Guérault (Antoine-M.). - La fromagerie devant les techniques \\ nouvelles, 1 vol. broché $15,5 \times 24,305$ pages. Editeur : S.E.P. \\ A.I.C., 42, rue du Louvre, Paris (1er). 1956. Prix : 2.200 franes, \\ franco: 2.320 franes.
}

Nous avons en son temps, salué, avec un grand intérêt, la parution des deux précédents ouvrages : L'industrie laitière : $1^{\circ}$ Organisation et Gestion des Entreprises; 20 Traitement et Fabrication, car nous étions persuadé qu'ils rendraient de très grands services aux techniciens et praticiens de l'industrie laitière.

Aujourd'hui, nous sommes heureux de présenter un livre d'importance au moins égale, mais basé sur des considérations un peu différentes qui nous semblent répondre tout à fait aux nécessités. En effet, comme l'écrit l'A., il ne s'agit pas d'une nouvelle édition, amendée et agrandie, mais bien d'un livre nouveau.

Le progrès scientifique est caractérisé en la matière par un certain nombre de notions nouvelles qui, pour servir les praticiens, doivent être interprétées, parfois condensées et parfois même expliquées, c'est-à-dire livrées sous une forme assimilable et immédiatement utilisable. Pour réussir une telle entreprise il fallait que s'en chargeât un spécialiste réunissant l'homme de science, l'industriel au plein sens du terme et le technicien-praticien averti. Sans le moindre sentiment de flatterie, nous ne craignons pas d'affirmer que sous ces conditions, il n'y avait de risque et que la sanction apparaîtra rapidement.

Après une introduction de mise au point et quelques généralités clairement énoncées, $A . M . G$, aborde immédiatement le sujet en traitant des rendements et mélanges, ce pourquoi il rappelle la définition du coefficient $G$.

Les chapitres suivants concernent les ferments et levains, la formation du caillé, le rôle du calcium dans la coagulation du lait par la présure, la coagulation eynérèse "égouttagè ".

La deuxième partie est consacrée aux fromages frais.

La troisième partie traite des fromages à pâte molle : fabrication, traitement après fabrication, maturation, les fromages à pâte molle (égouttage naturel et à caillé divisé).

Enfin, les importants chapitres de la quatrième partie sont réservés aux fromages à pâte pressée : fromage à pâte pressée non cuite (le Saint-Paulin pasteurisé, le Hollande), fromages à pâte pressée cuite (à partir de lait cru et à partir de lait pasteurisé).

Pour terminer, des pages, en annexes, mais non les moins importantes, contiennent des tableaux de rendements et mélanges.

Cet énoncé ne peut que très imparfaitement rendre compte de la qualité 